\title{
The Variable Intermittent Sampling Repeater Jamming Against Waveform Agile SAR
}

\author{
W.H. Yang \\ School of Electronic Science and Engineering, National \\ Univ. of Defense Technology, Changsha \\ Luoyang Electronic Equipment Test Center of China \\ Luoyang, China.
}

\author{
Y.G. Chen \\ School of Electronic Science and Engineering, National \\ Univ. of Defense Technology, Changsha \\ Beijing Institute of Tracking and Telecommunications \\ Technology, Beijing, China.
}

\begin{abstract}
A new type of jamming against waveform agile SAR is proposed, which is called the variable intermittent sampling repeater jamming. The jamming can form false vivid targets through repeating waveform agile SAR different subsection signal as design in the current pulse in the waveform agile SAR current pulses. So it may realize electronic counter measure against waveform agile SAR feasibly in practice. Detailed discussions about the jamming performance are given. The processing output of the jamming is derived theoretically. The theoretical feasibility is proved by simulation experiments.
\end{abstract}

Keywords-SAR; variable intermittent sampling; waveform agile; electronic counter measure

\section{INTRODUCTION}

Synthetic aperture radar (SAR) is an all weather imaging radar for strategic reconnaissance and battlefield surveillance systems, which makes it a sever threat to important military targets. Therefore the jamming technology for countering SAR has become an important issue in Electronic Counter Measure (ECM) area. Now /the active decoy jamming based on Digital Radio Frequency Memory (DRFM) could achieve high processing gain from coherent signals processing in [1-7]. But this traditional repeater jamming against SAR based on repeating the former time SAR pulse is difficult to quickly adapt to the large pulse width waveform agile SAR in $[3,4]$.

In order to handle this issue, the intermittent sampling jamming against SAR modulated in fast or slow time is studied in [4], but this jamming need modulation in time domain process to form area jamming images. So a new jamming is proposed, called the variable intermittent sampling repeater jamming, which is developed from the intermittent sampling jamming in [4,7], and it can form area jamming images without time domain process which is more feasible in practice. The jamming can repeat waveform agile SAR different subsection signal in the current pulse with little delay through the variable intermittent sampling repeating. Firstly, the model of the variable intermittent sampling repeater jamming is established for waveform agile SAR. Secondly, the jamming is imaged in the theory, and characteristics of the jamming imaging are analyzed in the waveform agile SAR. In the end, it is shown by the different SAR experiments that the variable intermittent sampling repeater jamming is simulated and imaged. The theory and imaging characteristics of the second part are verified by simulation experiments.

\section{THE MODEL OF THE VARIABLE INTERMITTENT SAMPLING REPEATER JAMMING}

It is well known that waveform agile SAR system should also utilize the two-dimension matched filtering. Suppose waveform agile SAR signal might vary chirp rate pulse, and it can be written as

$s\left(\hat{t}, t_{m}\right)=\operatorname{rect}\left(\frac{t-m T_{0}}{T_{p}(m)}\right) \exp \left[\mathrm{j} 2 \pi\left(f_{0} \hat{t}+F(m)\left(\mu_{r}+\gamma_{m}\right) \hat{t}^{2} / 2\right)\right]$

where $T_{p}(m)=T_{p} \mu_{r} /\left(\mu_{r}+\gamma_{m}\right)$ is LFM's the mth pulse duration width, $\mu_{r}+\gamma_{m}$ is the mth pulse chirp rate, $\mu_{r}$ is the chirp rate median, $\gamma_{m}$ is the $m$ th pulse chirp rate agility modulation, $f_{0}$ is carrier frequency, $\hat{t}$ is the fast time (in range), and $t_{m}$ is the slow time (in azimuth), $(m=0, \pm 1, \pm 2, \pm 3, \ldots), \quad \operatorname{rect}[t / T]= \begin{cases}1 & -T / 2 \leq t \leq T / 2 \\ 0 & \text { otherwise }\end{cases}$

\section{A. The Variable Intermittent Sampling Principle}

The principles of the variable intermittent sampling repeater jamming are as follows: The waveform agile SAR signal is variably sampled with high-fidelity intermittent, projecting to SAR as soon as possible. Then the next section variable signal is being variable intermittent sampled. So this jamming is received as the current pulse target echo being received. The jamming method for different pulses will do as method in this pulse. Then the variable intermittent sampling repeater jamming is formed.

The variable intermittent sampling function is developed from intermittent sampling in [4,7], it can be written as

$$
p(t)=\operatorname{rect}\left(\frac{\hat{t}}{T_{w}(t)}\right) \otimes \sum \delta\left(t-\sum T_{s}(t)\right)
$$

where $T_{s}(t)$ is the variable sampling duration width , $T_{w}(t)$ is the variable sample pulse width in $T_{s}(t), “ \otimes ”$ denotes convolution, $\delta(\cdot)$ is Dirac function.

Then SAR signal being sampled by variable intermittent sampling $s_{1}\left(\hat{t}, t_{m}\right)$ is written as 


$$
s_{1}\left(\hat{t}, t_{m}\right)=s\left(\hat{t}, t_{m}\right) \cdot p(t)
$$

The jamming delay in repeater can be controlled by the jammer in a short interval after sampling the SAR signal, and signal sampling center interval to the original signal simply delay time is usually a constant for the tone; as the intermittent sampling, this jamming can also be repeated directly after sampling, or be repeated several times being called duplication repeater.

Considered efficient of jamming power, the jammer is usually working in sample storage, or in jamming; So suppose $T_{s}(t) / T_{w}(t)=Q=1 / D$, the style of variable intermittent sampling may be identified: for the qth repeating delay time in duplication repeating, repeating jamming delay time from directly repeating sampling signal is $\tau_{r}(q), q=1,2 \cdots Q$.

The variable intermittent sampling repeater jamming can also be seen as containing modulation in fast time and in slow time domain. For simply in this paper, we can choose variable intermittent time as:

$$
T_{s}(t)=T_{s}(0)+2 r \cos \left(2 \pi f_{M} t\right) / c
$$

Where $\mathrm{c}$ is light speed, and variable setting is $r, f_{M}$, $T_{s}(0) \square 2 r / c$. In theory, this variable intermittent sampling repeater jamming will be imaged as the cosine modulation repeater jamming.

\section{B. The Model of Jamming}

The coordinate for SAR antenna phase center distance is $\left(v t, 0, H_{0}\right)$. The jammer coordinate is $\left(x_{J} t, y_{J}, H_{J}\right)$.

Then the distance between the jammer to SAR antenna phase center is:

$$
\begin{aligned}
R_{J}\left(t_{m}\right) & =\sqrt{\left(v t_{m}\right)^{2}+y_{J}^{2}+\left(H_{0}-H_{J}\right)^{2}} \\
& =\sqrt{\left(v t_{m}\right)^{2}+R_{J}^{2}} \approx R_{J}+\left(v t_{m}\right)^{2} / 2 R_{J}
\end{aligned}
$$

So the time for SAR to receive the qth repeating jamming signal

is:

$\tau_{k, q}=T_{w}(t)+2 R_{J}\left(t_{m}\right) / c+\tau_{r}(q)+\tau_{k}=\tau_{k q}^{\prime}+2 r \cos \left(2 \pi f_{M} t\right) / c$

where $\tau_{k q}^{\prime}$ can be seen as the $k^{\text {th }}$ tone jamming center for fast time delay.

The variable intermittent sampling repeater jamming signal will be received, and it can be expressed as

$$
\begin{aligned}
& \sum_{q} \sum_{k} s_{j q k}\left(\hat{t}, t_{m}\right)=\sum_{q} \sum_{k} \delta(k, m) s_{1}\left(\hat{t}-\tau_{k, q}, t_{m}\right) \\
& =\sum_{q} \sum_{k} \delta(k, m) s_{1}\left(\hat{t}-\tau_{k q}^{\prime}-2 r \cos \left(2 \pi f_{M} t\right) / c, t_{m}\right)
\end{aligned}
$$

where $\delta(k, m)$ is the ${ }^{\text {th }}$ tone signal amplitude for SAR receiving.

When jamming power can be seen as const, we can get the variable intermittent time as cosine moving the slow time modulation form in azimuth. The variable intermittent sampling modulation can be expressed as:

$$
\begin{aligned}
& \delta(k, m) \exp \left(\mathrm{j} 4 \pi r f_{0} \cos \left(2 \pi f_{M} t_{m}\right) / c\right)=\sum_{l=1}^{L} \delta_{k, l} \exp \left(\mathrm{j} 2 \pi \cdot \frac{-2 x_{k, l} f_{0} v t_{m}}{R_{J} c}\right) \\
& \propto \delta(k, m) \sum_{n=-\infty}^{\infty} J_{n}\left(4 \pi r f_{0} / c\right) \exp \left(j n 2 \pi f_{M} t_{m}\right)
\end{aligned}
$$

where $x_{k, l}$ can be seen as distribution point series in azimuth, $l=1 \cdots L$, and $J_{n}(x)$ is Bessel function.

\section{JAMMING IMAGE IN THE WAVEFORM AGILE SAR}

The variable intermittent sampling repeater jamming signal received by SAR is indeed subject to the same signal processing steps of the real target echo, and what may be influenced on the SAR imaging is interpreted as the output of the 2-D LFM the jamming match filtering. So jamming pulse compression is given in the following part.

Firstly, the tone 1 repeating directly variable intermittent sampling repeater jamming signal $s_{j 11}\left(\hat{t}, t_{m}\right)$ is matched filtering of fast time in the waveform agile SAR, this output is:

$$
\begin{aligned}
& y_{1}\left(\hat{t}, t_{m}\right)=s_{j 11}\left(\hat{t}, t_{m}\right) \otimes s\left(\hat{t},-t_{m}\right) / T_{p}(m) \\
& \approx \operatorname{rect}\left(\tau_{11}^{\prime} / 2 T_{p}(m)\right) \delta(1, m) D \sum_{n} \operatorname{sinc}(n D) \\
& \operatorname{sinc}\left(\left(\left(\mu_{r}+\gamma_{m}\right)\left(\hat{t}-\tau_{11}^{\prime}\right)\right)\left(T_{p}(m)-\left|\hat{t}-\tau_{11}^{\prime}\right|\right)\right) . \\
& \exp \left(-\mathrm{j} 2 \pi f_{0}\left(\tau_{11}+n /\left(T_{s}\left(t_{m}\right)\left(\mu_{r}+\gamma_{m}\right)\right)\right)\right.
\end{aligned}
$$

where $\tau_{11}$ is the fast time peak center. $(n=0, \pm 1, \pm 2, \pm 3, \ldots)$

It is shown that this jamming being matched filtering in fast time generates to the envelope convolution of the trigonometric functions as the target echo. And the variable intermittent sampling repeater jamming peak image focuses very well in fast time peak with variable range.

The variable intermittent sampling repeater jamming has the advantage of signal matched filter in fast time domain. And this imaging peak is proportional to the intermittent sampling duty cycle and the signal amplitude.

Next this jamming in waveform agile SAR is matched filtering in azimuth, the azimuth match filtering function is $h_{a}\left(t_{m}\right)=\operatorname{rect}\left(t_{m} / T_{L}\right) \cdot \exp \left(j 2 \pi f_{0}\left(v t_{m}\right)^{2} /\left(R_{J} c\right)\right), T_{L}$ is synthetic aperture time. So the output for jamming $s_{j 11}\left(\hat{t}, t_{m}\right)$ image $\mathbf{I}_{11}$ can be written as:

$$
\begin{aligned}
& \mathbf{I}_{11}=y_{1}\left(\hat{t}, t_{m}\right) \otimes h_{a}\left(t_{m}\right) \\
& \propto \sum_{l=1}^{L} \delta_{1, l} \operatorname{sinc}\left(\mu_{a}\left(\mu_{m}\left(\hat{t}-\tau_{11}^{\prime}\right)\right)\left(T_{p}(0)-\left|\hat{t}-\tau_{11}^{\prime}\right|\right)\right] . \\
&
\end{aligned}
$$

where the corresponding peak in the slow time is shown as the jamming point in azimuth being designed.

When the jamming can be seen as the cosine modulation repeater jamming, the output for image $\mathbf{I}_{11}$ can also be 
written as:

$$
\begin{aligned}
& \mathbf{I}_{11}=y_{1}\left(\hat{t}, t_{m}\right) \otimes h_{a}\left(t_{m}\right) \\
& \propto \sum_{n} J_{n}\left(4 \pi r f_{0} / c\right) \operatorname{sinc}\left(\mu_{a}\left(t_{m}-\frac{n f_{M} R_{J} c}{2 f_{0} v^{2}}\right)\left(T_{L}-\mid t_{m}-\frac{n f_{M} R_{J} c}{2 f_{0} v^{2}}\right)\right) . \\
& \operatorname{sinc}\left[\left(\mu_{r}\left(\hat{t}-\tau_{11}^{\prime}\right)\right)\left(T_{p}(0)-\left|\hat{t}-\tau_{11}^{\prime}\right|\right)\right]
\end{aligned}
$$

Of course, the whole jamming might be imaged to form false targets. The vivid targets $\mathbf{I}_{J}$ can be easily distinguished from the whole jamming imaging.

$$
\begin{aligned}
& \mathbf{I}_{J}=\sum_{q} \sum_{k} \mathbf{I}_{q k} \\
& \propto \sum_{q} \sum_{k} \sum_{n} J_{n}\left(4 \pi r f_{0} / c\right) \operatorname{sinc}\left(\mu_{a}\left(t_{m}-\frac{n f_{M} R_{J} c}{2 f_{0} v^{2}}\right)\left(T_{L}-\left|t_{m}-\frac{n f_{M} R_{J} c}{2 f_{0} v^{2}}\right|\right)\right) . \\
& \quad \operatorname{sinc}\left[\left(\mu_{r}\left(\hat{t}-\tau_{q k}^{\prime}\right)\right)\left(T_{p}(0)-\left|\hat{t}-\tau_{q k}^{\prime}\right|\right)\right]
\end{aligned}
$$

Suppose the jamming sequence is mimicked by DRFM repeat jammer $s\left(\hat{t}, t_{(m-1)}\right)$ or other sequence. The jamming will be suppressed by matching filtered, where the peak power of jamming imaging is about $20 \mathrm{~dB}$ below the focused target signal matching filtered in[3].

\section{THE SIMULATION EXPERIMENT}

The jamming power is constant, the variable intermittent sampling repeater jamming and traditional jamming based on repeating the former pulse is compared against waveform agile SAR and traditional SAR without waveform agility. SAR carrier frequency is $1 \mathrm{GHz}$, bandwidth is $100 \mathrm{MHz}$, and agility chirp rate $\max \left|\gamma_{m} / \mu_{r}\right|$ is 0 (SAR0 without waveform aglity) or waveform agile SAR1 0.6 ; azimuth beam angle is 3.6 degrees. SAR transmit power is $4.5 \mathrm{KW}$, antenna gain is $35 \mathrm{~dB}$.

The jammer power is $1 \mathrm{~W}$. And jammer antenna gain is $10 \mathrm{~dB}$, and jammer error mismatch is $5 \mathrm{~dB}$. We can choose the one-tone variable intermittent sampling repeater jamming, and the width of this jamming1 is $T_{s}(t)=1.1 \times 10^{-7}+2 \pi \cdot \cos (128 t) / c$, and $D=1 / 2$, and the traditional jamming 0 which sequence is based on repeating the former time SAR pulse is also cosine motion modulation with the same jammer power and center delay time $1.1 \times 10^{-7} \mathrm{~s}$, comparison to jamming 1 phase cosine modulation $\varphi\left(t_{m}\right)=2 \pi f_{0} \cos \left(128 t_{m}\right) / c$.

SAR antenna phase center distance coordinate along the track is $(100 t, 0,6000)$.The stationary jammer coordinate is $(0,8000,0)$. Firstly, the traditional cosine motion modulation jamming0 in SAR0 without waveform agility is simulated and imaged. The jammer and the cosine motion modulation jamming0 imaging area is shown as Fig.1(a), and the planning map $(x=0)$ in this image is shown as Fig. 1 (b). It proves that the jamming0 images well in SAR without waveform agility.

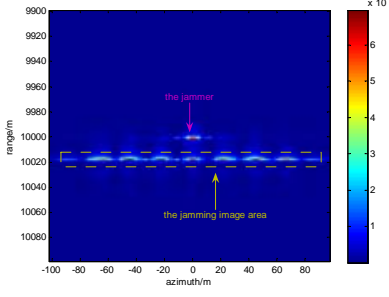

(a) SAR image

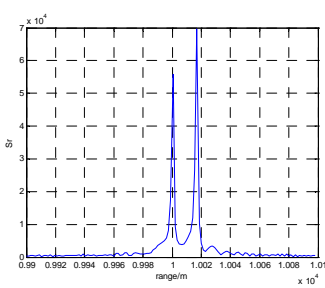

(b)The planning map $(x=0)$
FIGURE I. THE JAMMING0 IN SAR 0

Then the variable intermittent sampling repeater jamming1 in SAR0 without waveform agility is simulated. This image is shown as Fig.2(a), and the planning map $(x=0)$ in this image is shown as Fig.2 (b). It proves that this variable intermittent sampling repeater jamming images as the cosine motion modulation intermittent sampling repeater jamming in [4], so it effect well for SAR without waveform agility.

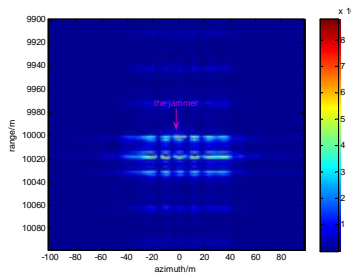

(a) SAR image

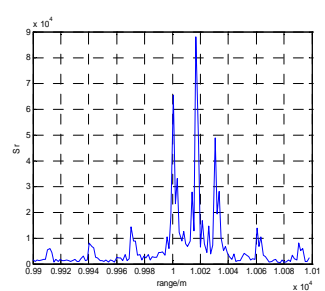

(b)The planning map $(x=0)$
FIGURE II. THE JAMMING1 IN SAR0

In waveform agile SAR1, the traditional cosine motion m3(a), and the planning map in this image is shown as Fig.3 (b). It shows the traditional jamming0 images nearly as noise clutter. So the traditional jamming will be suppressed by matching filtered, even without considering the SAR waveform agility "punishment" function processing as[3].

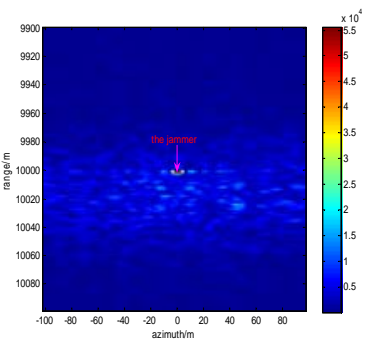

(a) SAR image

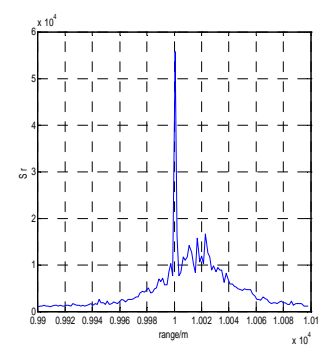

(b)The planning map $(x=0)$
FIGURE III. THE JAMMING0 IN SAR1

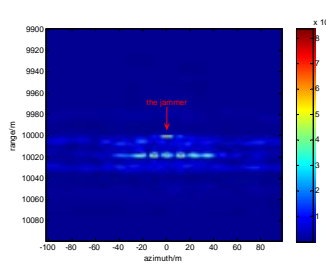

(a) SAR image

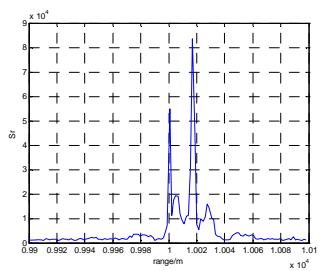

(b)The planning map $(x=0)$
FIGURE IV. THE JAMMING1 IN SAR 1 
In the same waveform agile SAR, the variable intermittent sampling repeater jamming1 is simulated. The image is shown as Fig.4(a), and the planning map in this image is shown as Fig.4 (b). It proves that the variable intermittent sampling repeater jamming can image well in jamming main peak area for waveform agile SAR, while other distance sideways lobe peak in SAR is decreased.

The variable intermittent sampling repeater jamming will be able to effect in other waveform agile SAR, which is rooted in the intermittent sampling jamming adapt to waveform agile SAR current pulse matched filter. The different intermittent sampling repeater styles jamming have been studied in [4], so we won't do it again here.

The variable intermittent sampling repeater jamming can also form vivid targets, which can be focused and form false phony targets in jamming main peak. The residual energy image, range delay and excursion in azimuth of jamming images accord with theoretical analysis.

This jamming technique is effective and feasible in practice, which has the prominent advantages against waveform agile SAR. The variable intermittent sampling repeater jamming can adapt to the waveform agile SAR current pulses. The jamming provides a new feasible approach against waveform agile SAR.

\section{REFERENCES}

[1] Sdg R.T Ekestorm, Christopher K. An all-digital image synthesizer for countering high-resolution radars [D].Naval Postgraduate School, Monterey, A 93943-5000, 2000.

[2] Wang W.Q, Cai J.Y. A Technique for jamming Bi- and Multistatic SAR Systems[J]. IEEE Geoscience and Remote Sensing Letters, 2007, 4(1):80 82.

[3] Mehrdad S., SAR-ECCM using phase-perturbed LFM chirp signals and DRFM repeat jammer penalization [J], IEEE TRANS ON AES VOL. 42, NO. 1 JANUARY 2006, 191 205.

[4] Yang W.H, Chen Y.G, Wang Tao. The Intermittent sampling jamming against SAR modulated in fast or slow time[J]. Joural of systems engineering and electronics 2012,12:2456 2462.

[5] Wang X.S, Liu J.C, Zhang W.M, et al. Mathematical principles of intermittent sampling repeater jamming [J]. Science in China, Series E: Information Sciences. 2006, 36(8):891 901(in Chinese).

[6] Yang W.H, Liu J., Wang T. the intermittent sampling scatter wave jamming against SAR[J]. Joural of Astronautics 2012,33(3):367 373.

[7] Wu X.F, Wang X.S, Lu H.Z. Study on intermittent sampling repeater jamming to SAR. Joural of Astronautics 2009,30(5):2043 2050 . 